Ferrata Storti Foundation

\title{
Gemtuzumab ozogamicin in children with relapsed or refractory acute myeloid leukemia: a report by Berlin-Frankfurt-Münster study group
}

\author{
Naghmeh Niktoreh,,$^{1^{*}}$ Beate Lerius, ${ }^{1 *}$ Martin Zimmermann, ${ }^{2}$ Bernd Gruhn, ${ }^{3}$ \\ Gabriele Escherich, ${ }^{4}$ Jean-Pierre Bourquin, ${ }^{5}$ Michael Dworzak, ${ }^{6}$ \\ Lucie Sramkova, ${ }^{7}$ Claudia Rossig, ${ }^{8}$ Ursula Creutzig, ${ }^{2}$ Dirk Reinhardt ${ }^{1}$ \\ and Mareike Rasche ${ }^{1}$
}

Haematologica 2018

Volume 104(1):120-127

\begin{abstract}
${ }^{1}$ Department of Pediatric Hematology and Oncology, University Hospital Essen, Germany; ${ }^{2}$ Department of Pediatric Hematology and Oncology, Hannover Medical School, Germany; ${ }^{3}$ Department of Pediatrics, Jena University Hospital, Germany; ${ }^{4}$ Department of Pediatric Hematology and Oncology, Eppendorf University Hospital, Hamburg, Germany; '5ivision of Pediatric Hematology/Oncology, University Children's Hospital Zurich, Switzerland; ${ }^{6}$ St. Anna Children's Hospital and Children's Cancer Research Institute, Department of Pediatrics, Medical University of Vienna, Austria; ${ }^{7}$ Department of Pediatric Hematology and Oncology, $2^{\text {nd }}$ Faculty of Medicine, Charles University and University Hospital Motol, Prague, Czech Republic and ${ }^{8}$ University Children's Hospital Münster, Pediatric Hematology and Oncology, Germany
\end{abstract}

${ }^{\star} N N$ and $B L$ contributed equally to this work as first authors.

\section{Correspondence: \\ mareike.rasche@uk-essen.de \\ Received: February 23, 2018. \\ Accepted: August 3, 2018. \\ Pre-published: August 31, 2018.}

doi:10.3324/haematol.2018.191841

Check the online version for the most updated information on this article, online supplements, and information on authorship \& disclosures: www.haematologica.org/content/104/1/120

\section{(c)2019 Ferrata Storti Foundation}

Material published in Haematologica is covered by copyright. All rights are reserved to the Ferrata Storti Foundation. Use of published material is allowed under the following terms and conditions:

https://creativecommons.org/licenses/by-nc/4.0/legalcode. Copies of published material are allowed for personal or internal use. Sharing published material for non-commercial purposes is subject to the following conditions:

https://creativecommons.org//icenses/by-nc/4.0/legalcode, sect. 3. Reproducing and sharing published material for commercial purposes is not allowed without permission in writing from the publisher.

\section{(c) (i) (s)}

\section{ABSTRACT}

$\mathrm{D}$ espite intensified salvage treatments, children with relapsed/refractory acute myeloid leukemia (AML) have poor survival. We evaluated gemtuzumab ozogamicin (CD33-targeted drug) used on a compassionate basis in patients diagnosed from 1995 until 2014 within Acute Myeloid Leukemia Berlin-Frankfurt-Münster studies, and identified 76 patients ( $<18$ years) with highly-advanced and pre-treated AML [refractory de novo acute myeloid leukemia $(n=10)$, de novo AML refractory to relapse ( $1^{\text {st }}$ early: $n=41 ; 1^{\text {st }}$ late: $n=10 ; 2^{\text {nd }}$ or more: $n=10)$, and secondary AML ( $n=5)]$. At doses of $2.5-10 \mathrm{mg} / \mathrm{m}^{2}$, gemtuzumab ozogamicin was administered in 1-4 cycles as single agent $(47 \%)$, combined with cytarabine $(47 \%)$, or others $(6 \%)$. Most common grade $3 / 4$ adverse events were infections or febrile neutropenia $(78 \%$ of severe adverse events), infusion-related immunological reactions (6\%), and gastrointestinal symptoms (5\%). Three patients experienced venoocclusive disease (one fatal due to exacerbation of a pre-existing cardiomyopathy). Sixty-four percent received subsequent hematopoietic stem cell transplantation. Probability of 4-year overall survival was $18 \pm 5 \%$ in all, $27 \pm 7 \%$ in patients with and $0 \%$ in patients without hematopoietic stem cell transplantation $(P<0.0001)$. Administration of gemtuzumab ozogamicin on a patient-specific, compassionate use basis was frequently considered in our study group and proved to be effective for bridging children with very advanced AML to hematopoietic stem cell transplantation. Uniform prospective studies for these patients are urgently needed.

\section{Introduction}

Treatment of acute myeloid leukemia (AML) in children has improved remarkably during the past decades; however, pediatric patients with relapsed and refractory AML still have poor outcomes. ${ }^{1-6}$ These outcomes rely on disease-dependent characteristics, such as initial cytogenetics, in addition to response-to-therapy-related factors like the interval between initial diagnosis and relapse. ${ }^{7-10}$ Considering poor outcome and high toxicity of current salvage therapies, new targeted molecular treatments are needed. ${ }^{11-13}$

Gemtuzumab ozogamicin (GO) is an immunotoxin consisting of a potent humanized monoclonal antibody against $\mathrm{CD} 33$, and targets $\mathrm{CD} 33$ positive cells 
which are present in approximately $80-90 \%$ of childhood AML. ${ }^{14,15}$ Although CD33 has been considered as a specific marker for hematopoietic cells of the myeloid lineage for a long time, this surface marker is also found to be expressed in hepatocytes which may potentially cause some off-target effects. ${ }^{16}$

Treatment with GO in different settings has previously been shown to be of value in pediatric AML. The first international experience of treatment with GO as monotherapy for compassionate use for children with relapsed or refractory AML $(n=15)$ in 2003 suggested the efficacy of this treatment with doses of $4-9 \mathrm{mg} / \mathrm{m}^{2}$ in up to 3 cycles. ${ }^{17}$ A later phase II study showed that treatment with two doses of $7.5 \mathrm{mg} / \mathrm{m}^{2}$ GO with 14-day intervals in children with advanced AML (refractory, first refractory relapse or $\geq$ second relapse) led to significantly higher survival in patients who received GO compared to patients who did not receive this treatment (3-year probability of overall survival: $27 \%$ vs. $0 \%$, respectively; $P=0.001) .{ }^{18}$ In addition to monotherapy, a good response rate was also achieved when GO was administered in combination with cytarabine in 17 children with relapsed or refractory AML [overall complete remission (CR) rate: $53 \%{ }^{19}$

Currently there are at least 9 active clinical trials worldwide investigating the effect of GO in AML (de novo, relapsed, or refractory). One of these trials only recruits children and 4 are recruiting children in addition to adults or elderly patients (Online Supplementary Table S1). Of interest, there is one active clinical trial which studies treatment with GO in compassionate use in refractory or relapsed AML (clinicaltrials.gov identifier: 02312037), which recruits children, adult, and elderly groups of patients. ${ }^{20}$

Considering the above-mentioned challenges in the treatment of relapsed and refractory pediatric AML, the infrequency of new treatment options, and in addition, the restricted accessibility of GO, we aimed to identify patients treated with GO as compassionate use in the AML-Berlin-Frankfurt-Münster (AML-BFM) study group and to evaluate the efficacy and safety in this heavily pretreated group of patients.

\section{Methods}

\section{Patients}

Between January 1995 and March 2014, 2601 children with initial diagnosis at the age of $\leq 18$ years were documented within the AML-BFM study group. Patients or guardians provided written informed consent. The current analysis was performed in accordance with the Declaration of Helsinki and was approved by the local ethics committee. The AML-BFM Study Group centrally reviewed the diagnosis of initial disease or relapse via bone marrow morphology and flow cytometry. All patients' records were evaluated retrospectively for the use of GO. Medical reports of the patients treated with GO were reviewed retrospectively for evaluation of treatment outcomes and adverse events (AE).

\section{Treatment protocols}

Before administration of $\mathrm{GO}$, patients were treated based on randomized, phase III studies AML-BFM 93, 98, and 2004 running in 75 centers in Germany, Austria, Switzerland, and the Czech Republic. These treatment protocols have been previously described in detail. ${ }^{21-23}$ All studies were performed after the approval by national ethics committees and institutional review boards. The AML-BFM 93 recruited patients between January
1993 and June 1998, and was followed by AML-BFM 98 which was opened in July 1998 and closed in June 2003. Between July 2003 and April 2004, the AML-BFM 98 Interim Study continued treatment of recruited patients with the best arm of the AML-BFM 98 trial. The AML-BFM 04 study was opened in April 2004 and randomization continued until April 2010. After April 2010 and until February 2014, the AML-BFM 2004 Interim Study continued treatment of patients with the experimental arm of the AML-BFM 2004 trial. Second-line treatment of patients included the Relapse AML 2001/01 trial ${ }^{24}$ which recruited patients from November 2001 to April 2009 and the International Registry Relapsed AML 2009.? Patients included in the current cohort had received intensive treatment and/or HSCT before administration of GO. Considering the first treatment after initial diagnosis as first attempt, and each following treatment block or HSCT as further individual attempts, most of the patients $(n=35,46 \%)$ in the total cohort received GO as their $3^{\text {rd }}$ treatment attempt (Online Supplementary Figure S1).

\section{Treatment with GO}

During the period of analysis, 217 patients with non-response (NR) and 654 patients with at least one event of relapse were documented (Online Supplementary Figure S2). Within these patient records, 98 (from 39 different centers) were found with a positive history of GO treatment, 10 of which were included in the previously mentioned phase II trial ${ }^{18}$ and who were, thus, excluded from the current study. The remaining 88 patients received $\mathrm{GO}$ on a compassionate use basis, after failure of their first- and/or second-line treatments and/or when the general condition of patients was so poor that further intensive chemotherapy was not possible. Treatment with GO in these patients was recommended by the centralized study co-ordination office, and finally prescribed by the patients' treating physicians at each local center. Patients or guardians provided written informed consent. GO was provided by International Pharmacy (San Francisco, CA, USA) through Clinigen (London, UK) and, for some patients, by Pfizer (New York, NY, USA) on a compassionate use program. From these 88 patients, 76 had sufficient data to evaluate the outcomes of GO use and were included in this study (Online Supplementary Figure S2). Three of the 76 patients have been previously reported. ${ }^{17}$ Grading of toxicity and adverse events was carried out using the NCI Common Terminology Criteria for Adverse Events (CTCAE), version 4.03, revised in June 14, 2010. ${ }^{25}$ Records concerning the safety evaluation of GO were not available for 5 patients (Online Supplementary Figure S2).

\section{Definitions and statistical analysis}

Bone marrow (BM) relapse was defined by presence of $>5 \%$ of leukemic cells in the BM. Relapse events during the first 12 months from diagnosis of AML were considered as early relapse. ${ }^{3}$ Complete remission (CR) was defined with the presence of less than $5 \%$ blasts in morphological examination of BM in addition to satisfactory hematologic recovery (absolute neutrophil count $>1.0$ $\mathrm{x} 10^{9} / \mathrm{L}$, platelet count $>80 \times 10^{9} / \mathrm{L}$ in the peripheral blood). ${ }^{21}$ Reaching CR without complete blood recovery was defined as incomplete CR (CRi). ${ }^{21}$ Response to GO was evaluated in patients who received HSCT after treatment with GO and was defined as CR plus CRi (CR/CRi). Persistence of $\geq 5 \%$ BM leukemic blasts was categorized as NR.

Overall survival (OS) after GO was defined as the time from the first dose until death by any cause or until the last follow up. Probability of OS was calculated according to Kaplan-Meier and compared by log-rank test. $P<0.05$ was considered significant. Data were analyzed using SAS (Statistical Analysis System Version 9.4; SAS Institute, Cary, NC, USA). Data acquisition was stopped on $1^{\text {st }}$ June 2017. 


\section{Results}

Patients' characteristics and treatment with GO

The majority of patients were initially diagnosed with AML with the French-American-British (FAB) classification of M4 (without atypical eosinophils) or M5 ( $n=29$,
$39 \%)$ and $13(17 \%)$ patients had white blood cell (WBC) counts higher than $100 \times 10^{\circ} / \mathrm{L}$ at the time of their diagnosis. In total, $67(88 \%)$ patients had high-risk AML as defined by morphology, cytogenetics and response to treatment, retrospectively (Table 1). Most of the patients $(n=43,56 \%)$ had been previously treated with liposomal

Table 1. Patients' characteristics at initial diagnosis.

$\begin{array}{lcc}\text { Feature } & \text { n } & \% \\ \text { Total number of patients } & 76 & 100 \\ \text { Age, years } & & \\ \text { Median (range) } & & \\ \text { Categories } & & \\ 0-2 & 15 & 20 \\ 3-10 & 26 & 34 \text { months - } 17 \text { years }) \\ 11-18 & 35 & 46 \\ \text { Sex } & & \\ \text { Female } & 33 & 43 \\ \text { Male } & 43 & 57\end{array}$

FAB Classification

$\begin{array}{lcc}\text { M0 } & 6 & 8 \\ \text { M1/M2 } & 25 & 33 \\ \text { M3 } & 1 & 1 \\ \text { M4E0-M5 } & 29 & 39 \\ \text { M6 } & 1 & 1 \\ \text { M7 } & 7 & 9 \\ \text { AML-not classified } & 7 & 9\end{array}$

WBC count at diagnosis

Median x10\%(range)

$14.8(0.39-324)$

Patients with $\mathrm{WBC} \leq 100 \times 10^{\circ} / \mathrm{L}$

$62 \quad 82$

Patients with $\mathrm{WBC}>100 \times 10^{\circ} / \mathrm{L}$

$13-17$

No data

1

Previous treatments

Pre-treatment with FLA/G

Pre-treatment with FLA/G+DX (+/- FLA/G) ${ }^{a}$

$12 \quad 16$

Pre-treatment without FLA/G+DX or FLA/G

HSCT prior to $\mathrm{GO}$ treatment

$\begin{array}{lll}\text { Yes } & 14 & 18 \\ \text { No } & 62 & 82\end{array}$

Disease status prior to $\mathrm{GO}$

Refractory de novo $\mathrm{AML}^{\mathrm{b}}$

De novo AML- refractory to ${ }^{1^{\text {st }}}$ early relapse

10

De novo AML-refractory to $1^{\text {st }}$ late relapse

$41-54$

De novo AML- refractory to $2^{\text {nd }}$ relapse

De novo AML-refractory to $3^{\text {rd }}$ relapse $^{c}$

Secondary AML- refractory to $1^{\text {st }} / 2^{\text {nd }}$ relapse ${ }^{c}$

Risk group ${ }^{\mathrm{d}}$

Standard risk

67

High risk

1

6

28

18 82

n: number; FAB classification: French-American-British classification; M4Eo-: AML M4 subtype without the presence of atypical eosinophils; AML: acute myeloid leukemia; HSCT: hematopoietic stem cell transplantation; FLA/G: fludarabine, cytarabine with or without granulocyte colony stimulating factor; DX: liposomal daunorubicin; GO: gemtuzumab

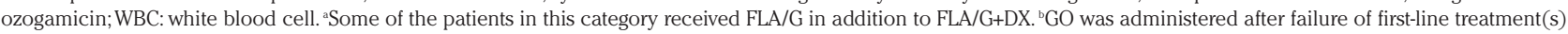
${ }^{\mathrm{C}} \mathrm{GO}$ was administered after failure of treatment for the relapse episode. ${ }^{\mathrm{S} S t a n d a r d}$ risk indicates FAB M1/2 with Auer rods, FAB M4 with atypical eosinophils (M4Eo), FAB M3 and/or favorable cytogenetics, such as t(8;21) and/or AML1-ETO, t(15;17), and/or PML-RARA and inv(16) or t(16;16) and/or CBFB/MYH1, if there was no persistence of BM blasts $(\geq 5 \%)$ on day 15 . FLT3-ITD positivity was not considered. All others were classified as high-risk patients. 
daunorubicin and fludarabine in addition to cytarabine with or without granulocyte colony stimulating factor (FLA/G+DX). Fourteen (18\%) patients received HSCT prior to treatment with GO (Table 1). GO was administered after failure of treatment attempts at different time points of therapy and most $(n=41,54 \%)$ of the patients were treated with $\mathrm{GO}$ after failure of treatment(s) for firstearly relapse of de novo AML (de novo AML, refractory to first early relapse) (Table 1$)$. GO was equally prescribed as either monotherapy $(n=36,47 \%)$ or in combination with cytarabine ( $n=36,47 \%)$, and the remaining patients $(n=4$, $6 \%$ ) received GO in combination with other agents (Table $2)$. Most patients $(n=48,63 \%)$ received one cycle of $\mathrm{GO}$ as monotherapy or combination therapy (Table 2). GO was frequently administered at doses of $3 \mathrm{mg} / \mathrm{m}^{2}$ or lower $(\mathrm{n}=37,49 \%)$ (Table 2$)$ and most of these patients received

Table 2. Details and outcomes of treatment with gemtuzumab ozogamicin.

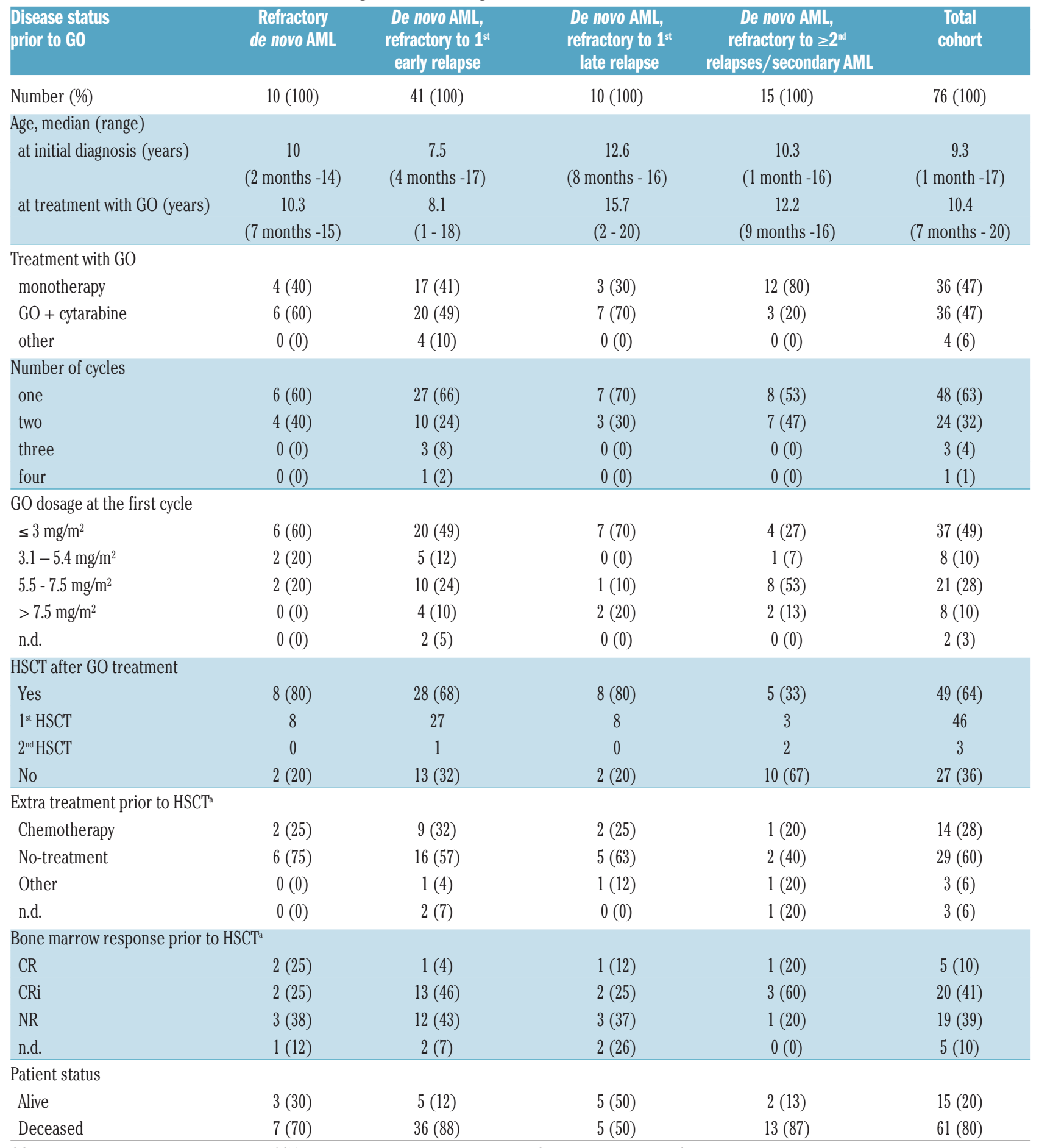

GO:gemtuzumab ozogamicin;n.d.:no data;HSCT: hematopoietic stem cell transplantation; CR: complete remission; CRi: complete remission with incomplete hematologic recov-

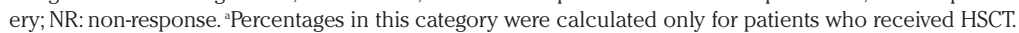


$\mathrm{GO}$ in combination with cytarabine or other agents following the failure of treatment after first early relapse ( $n=20$ of $37,54 \%$ ) (data not shown). When GO was administered as monotherapy, higher doses were used (Figure 1).

\section{Safety and toxicities}

Among 71 patients who were evaluated for AEs during the first cycle of treatment, most common AEs were grade $3 / 4$ infections or febrile neutropenia (61 events in 49 patients, 69\%) (Table 3). Two patients suffered from sepsis and were successfully managed with supportive and anti-bacterial therapy. Gastrointestinal (GI) symptoms (11 grade $1 / 2$ events in 11 patients and 4 grade $3 / 4$ events in 4 patients) and immunologic reactions such as infusionrelated fever, chills, or hypotension ( 7 grade- $1 / 2$ events in 7 patients and 5 grade $3 / 4$ events in 5 patients) were most frequently observed after infections. Due to interference with other treatments, GI events could not be assigned to GO with certainty.

Among grade 3 and 4 AEs, febrile neutropenia was most frequently observed in both groups of patients with monotherapy and combination therapy as well as in all different groups of patients independently of their previous treatments (FLA/G or FLA/G+DX or patients with HSCT prior to GO) (data not shown). The majority (4 of 5) of patients with severe infusion-related immunological reactions to $\mathrm{GO}$ treatment, had previously received $\mathrm{GO}$ as monotherapy with higher doses compared to patients with combination therapy (data not shown).

Veno-occlusive disease (VOD) occurred in 3 patients who received $\mathrm{GO}$ as monotherapy (one cycle) at doses of $6,7.5$, and $9 \mathrm{mg} / \mathrm{m}^{2}$. All 3 patients had FLA/G+DX as treatment before $\mathrm{GO}$, and received defibrotide as prophylaxis against VOD. Two of these patients were treated with HSCT prior to treatment with GO $\left(6\right.$ or $\left.7.5 \mathrm{mg} / \mathrm{m}^{2}\right)$. The patient treated with $6 \mathrm{mg} / \mathrm{m}^{2} \mathrm{GO}$ had a previous history of VOD and developed a GO-related VOD before the scheduled HSCT that was successfully treated without late effects or events. The other 2 patients could not receive HSCT after treatment with GO and both died. Cause of death in one of them was disease (leukemic) pro-

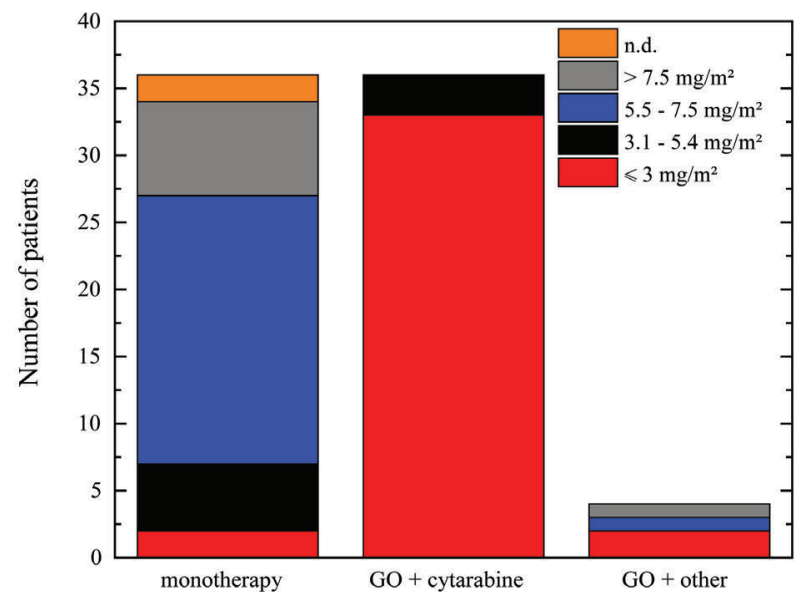

Figure 1. Gemtuzumab ozogamicin (GO) dosage. GO dosage based on administration with cytarabine or other agents. AML: acute myeloid leukemia; n.d. : no data. gression, and the other patient experienced exacerbation of a pre-existing cardiomyopathy leading to death 24 days after GO treatment. In addition, treatment with GO resulted in exacerbation of previous symptoms in 2 patients (pulmonary aspergillosis infection or gastrointestinal toxicity) and, due to overlap with other treatments and HSCT, the consequences of these AEs could not be exclusively correlated to GO (Table 3). Similarly, the consequences of different AEs in one patient with GO-related cytokine syndrome and pre-existing respiratory distress could not be distinguished from each other (Table 3). The respiratory distress in this patient was related to an aplasia-associated pneumonia which was caused by teatments before $\mathrm{GO}$.

\section{Outcome}

With a median follow up time of 4.3 years (range: $1-5$ years), the probability of 4 -year OS after treatment with
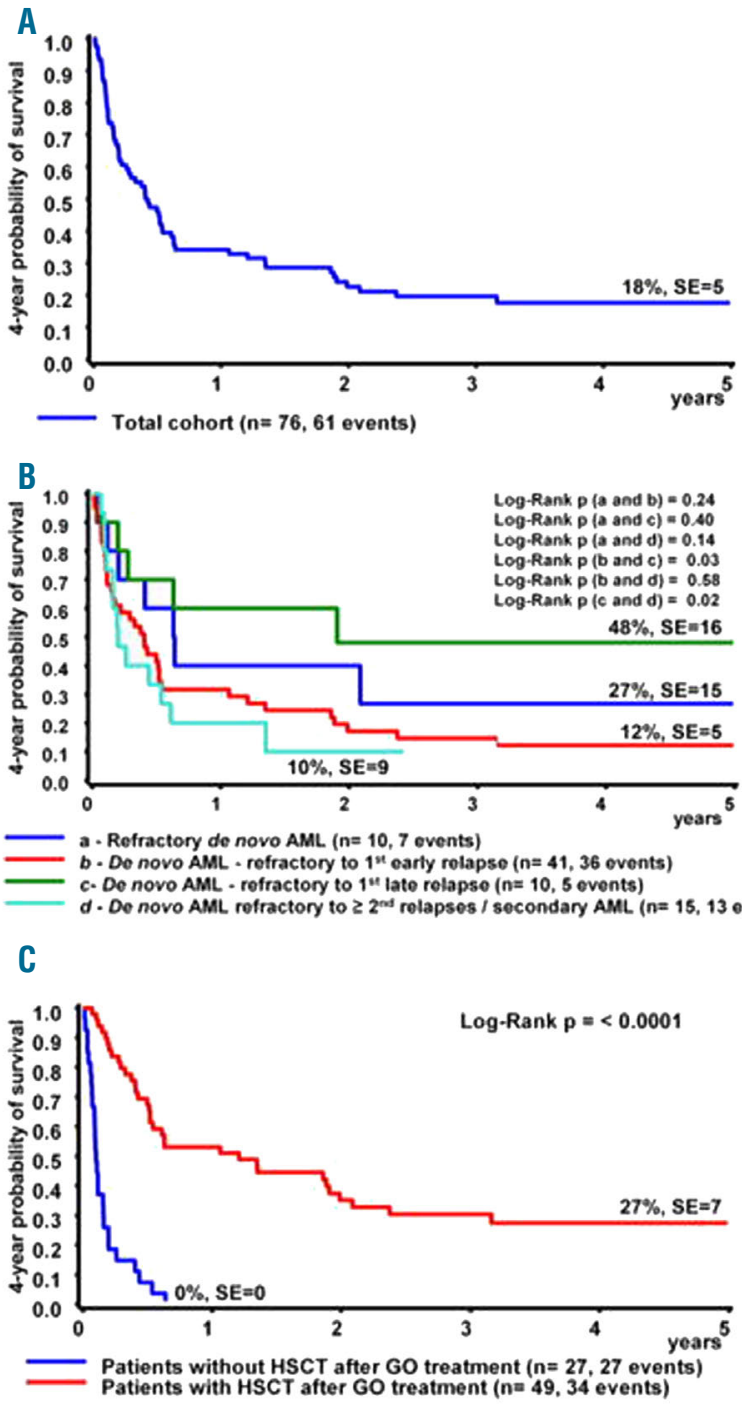

Figure 2. Survival after gemtuzumab ozogamicin (GO). (A) 4-year probability of overall survival (OS) in all patients. (B) 4-year probability of OS in different disease statuses. (C) 4-year probability of OS based on administration of hematopoietic stem cell transplantation (HSCT). pOS: probablity of OS; n: number of patients; AML: acute myeloid leukemia; SE: Standard Error. 
$\mathrm{GO}$ was $18 \pm 5 \%$ in the total cohort (Figure $2 \mathrm{~A}$ ). A comparison of disease status prior to treatment with $\mathrm{GO}$ between patient groups showed that patients who received GO after de novo AML refractory to first late relapse had a probability of 4 -year OS of $48 \pm 16 \%$; this is significantly higher than in patients with de novo AML refractory to first early relapse (probability of 4-year OS: $12 \pm 5 \%$; $P=0.03$ ) and patients with de novo AML refractory to second or more relapses/secondary AML (probability of 4-year OS: $10 \pm 9 \% ; P=0.02$ ) (Figure 2B). Based on the current retrospective analysis, factors such as number of cycles and administration of $\mathrm{GO}$ as monotherapy or in combination with cytarabine or other agents did not have any significant influence on survival (Online Supplementary Figure $S 3 A-C)$.

In the total cohort of patients, 27 (36\%) did not receive HSCT after treatment with GO (Table 2) and none of these patients survived (Figure 2C). Of note, most (10 of $15,67 \%$ ) patients with de novo AML refractory to second or more relapses/secondary AML, did not receive an HSCT after GO treatment; this is higher than in other groups of patients (Table 2).

From the total group of 76 patients, 49 (64\%) received HSCT after GO treatment and their probability of 4-year OS was $27 \pm 7 \%$. Thirty (61\%) patients with HSCT received GO in combination with cytarabine (data not shown). HSCT after GO was the first HSCT for most of the patients ( $n=46$, $94 \%$ ), and mean time to transplantation after GO treatment was $41 \pm 30$ days (range 11-135 days). The probability of 4year OS in patients who received HSCT early during the first three weeks after GO treatment was $9 \pm 9 \%$ and patients who received HSCT between three and six weeks after GO administration had a 4 -year OS of $40 \pm 11 \%$ $(P=0.06)$ (Online Supplementary Figure S3D).

Previous treatment with or without FLA/G+DX before $\mathrm{GO}$ administration had no influence on the percentage of
HSCT achieved after treatment with GO (FLA/G+DX group: 29 of 43, 67\%; no FLA/G+DX or FL/A group: 14 of $21,67 \%$ ) (data not shown). However, among transplanted patients, fewer patients with previous FLA/G+DX (7 of $29,24 \%$ ) survived compared to the patients without $\mathrm{FLA} / \mathrm{G}+\mathrm{DX}$ or FL/A (6 of 14, 42\%) (data not shown). In addition, one patient with de novo AML-M3 refractory to first late relapse is alive after treatment with GO followed by additional chemotherapy and HSCT.

Among 49 patients with subsequent HSCT, 25 (51\%) reached CR/CRi before HSCT (Table 2). Out of 19 patients with no response to GO before HSCT, $8(42 \%)$ received additional chemotherapy before HSCT and 3 of them survived. The remaining 11 (58\%) patients with no response, received HSCT with no further chemotherapy and 2 of these patients survived (data not shown).

\section{Discussion}

The major goal in the treatment of relapsed/refractory pediatric AML is to develop new therapeutic options to achieve complete remission and proceed to HSCT thereafter. $^{26}$ Considering the previous history of intensive chemotherapy and/or previous HSCT in these patients, novel treatment options should be efficient, but must also have acceptable toxicity profiles. However, the introduction of new drugs is challenging. Due to increasing regulatory challenges and requirements, as well as limited accessibility of drugs such as GO, it is more and more time consuming to open phase III trials. Hence a follow-up trial, that has been planned since 2009 after the end of the phase II trial, ${ }^{18}$ was not opened until September 2016 and recruited the first patient in August 2017 (EudraCT n.: 2010-018980-41)

In the current analysis, we have evaluated the use of GO

Table 3. Non-hematologic adverse events in 71 patients after the first treatment cycle with gemtuzumab ozogamicin.

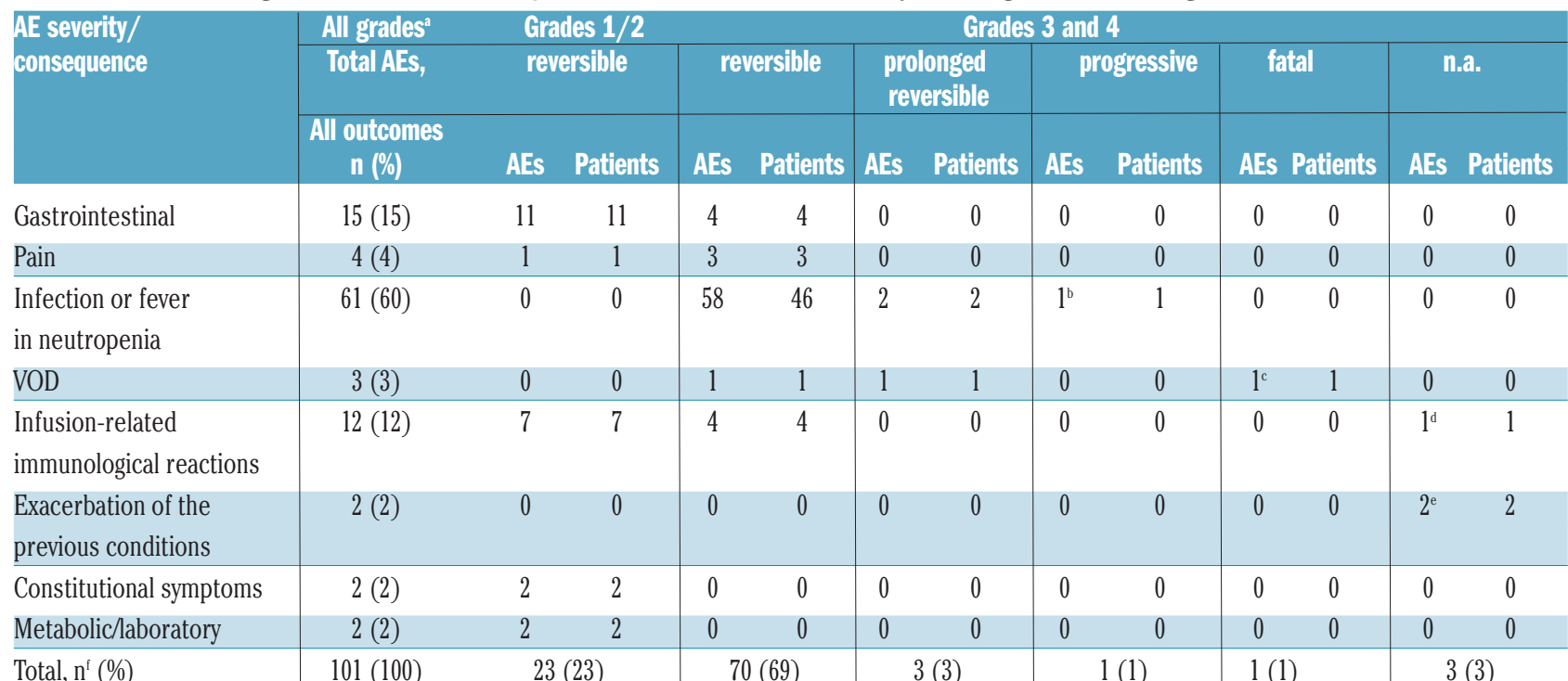

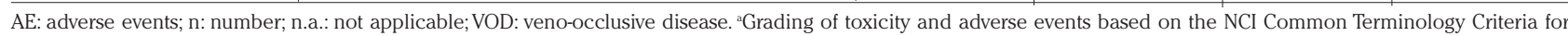

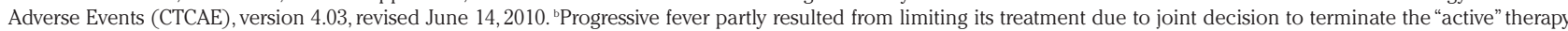
of the patient. 'GO-related VOD led to exacerbation of a pre-existing cardiomyopathy causing death 24 days after the treatment with GO. ${ }^{\mathrm{d}}$ Treatment with GO resulted in occurrence of cytokine syndrome in a patient with pre-existing respiratory distress due to aplasia-associated pneumonia. Following a decrease in level of consciousness, this patient died five days after occurrence of the cytokine syndrome. Treatment with GO resulted in worsening of previously present pulmonary aspergillosis infection or in worsening of previously observed gastrointestinal toxicity. ${ }^{\prime}$ All adverse events in 71 patients were evaluated. 
on a compassionate use basis in a cohort of children with refractory de novo AML and de novo or secondary AML refractory to relapse therapy. The use of GO in heavily pre-treated patients translated into a substantial rate of subsequent HSCT of $64 \%$ and a probability of 4-year OS of $27 \%$ in these patients. The safety profile in our cohort was tolerable, which may allow the use of GO in patients with a history of intensive therapies.

We have identified 88 and analyzed 76 children with relapsed/refractory AML who were treated with GO on a compassionate use basis. Compared to other studies which evaluated the outcomes of treatment with GO in patients with relapsed/refractory AML either on a compassionate use basis (Zwaan et al., 15 patients; ${ }^{17}$ Brethon et al., 17 patients) ${ }^{19}$ or with other treatment strategies (29 patients with relapsed/refractory AML in an open-label, dose-escalation study; ${ }^{27} 45$ children with relapsed/refractory AML included in the AAML00P2 randomized clinical trial $;^{28} 30$ children with advanced relapsed/refractory AML in an investigator-initiated phase II study) ${ }^{18}$ the current cohort contains the largest number of patients.

With GO doses of $2.5-10 \mathrm{mg} / \mathrm{m}^{2}, 64 \%$ of the patients included in our cohort subsequently received HSCT, and $51 \%$ of these patients reached complete remission with or without hematologic recovery before receiving HSCT. In 2005, Arceci et al. studied the effect of treatment with GO as a single agent with doses of $6-9 \mathrm{mg} / \mathrm{m}^{2}$ (2 doses, 2-week intervals) in 10 children with refractory AML and 19 with relapsed AML. ${ }^{27}$ They defined response to GO as achieving CR (presence of $\leq 5 \%$ blasts in BM with full hematologic recovery: hemoglobin level $\geq 9 \mathrm{~g} / \mathrm{dL}$, absolute neutrophil count $\geq 1.5 \times 10^{9} / \mathrm{L}$, and platelet count $\geq 100 \times 10^{9} / \mathrm{L}$ ) and showed a response rate of $30 \%$ and $26 \%$ in patients with refractory and relapsed AML, respectively, accompanied by acceptable safety profiles. ${ }^{27}$ In addition, in a study by Brethon et al., in 2006, outcome of monotherapy with $\mathrm{GO}$ on a compassionate basis (single dose: $3-9 \mathrm{mg} / \mathrm{m}^{2}$ ) in children with refractory (3 patients) and relapsed ( 9 patients) AML was investigated. ${ }^{29}$ The results of this study showed a CR (plus CR without hematologic recovery) rate of $25 \%$ in patients with subsequent $\mathrm{HSCT}^{29}$ Of note, a subsequent study by the same group in 2008 showed that the response rate was higher (overall CR: 53\%) when $\mathrm{GO}$ was administered in combination with cytarabine in children with relapsed or refractory AML. ${ }^{19}$ We could not confirm the benefit of this combination treatment in our cohort since combination of $\mathrm{GO}$ with cytarabine alone, or with cytarabine along with other agents or combination of GO with vincristine had no survival advantage compared to single agent therapy.

Myelosuppression accompanied by fever was the most common GO-related $\mathrm{AE}$ in our patients (49 events in 58\% of patients) (data not shown) that appeared to be independent of GO dosage, combination/monotherapy, previous treatments, or disease status prior to GO. Rates of myelosuppression by $\mathrm{GO}$ were previously reported at a range of $25-100 \%$ in different studies. ${ }^{19,28,29}$

In our cohort, the rate of VOD after treatment with $\mathrm{GO}$ was $4 \%$. Frequency of VOD related to monotherapy or combination GO therapy in children has been previously reported with a wide range across different studies from no events ${ }^{29,30}$ to $10-24 \% .^{27,28,31}$ However, taken together, the results of previous studies show that the incidence of $\mathrm{VOD}$ is directly associated with the absolute single dose of $\mathrm{GO}$, which is higher when it is administered as monotherapy. ${ }^{32}$ These findings are in accordance with the results of the current cohort. Considering the suggested increased risk of VOD in cases of monotherapy with GO, and the lack of a survival advantage between monotherapy and combination therapy in our current cohort, administration of $\mathrm{GO}$ in combination with other agents, such as cytarabine, may be useful to prevent this complication.

It should be noted that the current analysis is limited by its retrospective study design and especially by the lack of a control group. These restrictions have hindered our efforts to identify the contribution of compassionate treatment with GO as a single factor to the outcomes of the current study.

In conclusion, we have shown in a large cohort of patients with relapsed/refractory de novo and secondary AML, with a history of very intensive treatments including chemotherapy and/or HSCT, that administration of $\mathrm{GO}$ on a compassionate use basis was frequently considered. The study provides evidence that GO can enable a subsequent blast reduction that allowed HSCT in these patients and survival without imposing major adverse events. Since addition of GO showed the potential to improve treatment outcomes in the current cohort of patients with relapsed/refractory pediatric $\mathrm{AML}$, the role of GO in these patient groups should ideally be proven in large prospective randomized clinical trials. Therefore, we have now included $\mathrm{GO}$ as front-line treatment of relapsed/refractory pediatric AML in our ongoing phase III multicenter clinical trial (EudraCT number: 2010-01898041, recruiting since August 2017).

\section{Acknowledgments}

We are grateful to all patients who recruited in the corresponding $A M L-B F M$ trials and physicians and study staff involved in patient care and studies maintenance. We thank Mr. Jans Enno Mueller for his valuable contribution to the study data-base. Supplementary information is available at Hematologica's website.

\section{References}

1. Zwaan CM, Kolb EA, Reinhardt D, et al. Collaborative Efforts Driving Progress in Pediatric Acute Myeloid Leukemia. J Clin Oncol. 2015;33(27):2949-2962

2. Kaspers G. How I treat paediatric relapsed acute myeloid leukaemia. Br J Haematol. 2014;166(5):636-645.

3. Sander A, Zimmermann M, Dworzak M, et al. Consequent and intensified relapse therapy improved survival in pediatric AML: results of relapse treatment in 379 patients of three consecutive AML-BFM trials. Leukemia. 2010;24(8):1422-1428.

4. Horton TM, Perentesis JP, Gamis AS, et al. A Phase 2 study of bortezomib combined with either idarubicin/cytarabine or cytarabine/etoposide in children with relapsed, refractory or secondary acute myeloid leukemia: a report from the Children's Oncology Group. Pediatr Blood Cancer. 2014;61(10):1754-1760.

5. Nakayama H, Tabuchi K, Tawa A, et al.
Outcome of children with relapsed acute myeloid leukemia following initial therapy under the AML99 protocol. Int J Hematol. 2014;100(2):171-179.

6. Cooper TM, Alonzo TA, Gerbing RB, et al. AAML0523: a report from the Children's Oncology Group on the efficacy of clofarabine in combination with cytarabine in pediatric patients with recurrent acute myeloid leukemia. Cancer. 2014; 120(16):2482-2489.

7. Kaspers GJ, Zimmermann M, Reinhardt D, 
et al. Improved outcome in pediatric relapsed acute myeloid leukemia: results of a randomized trial on liposomal daunorubicin by the International BFM Study Group. J Clin Oncol. 2013;31(5):599-607.

8. Gorman MF, Ji L, Ko RH, et al. Outcome for children treated for relapsed or refractory acute myelogenous leukemia (rAML): a Therapeutic Advances in Childhood Leukemia (TACL) Consortium study. Pediatr Blood Cancer. 2010;55(3):421-429.

9. Webb DK. Management of relapsed acute myeloid leukaemia. Br J Haematol. 1999;106(4):851-859.

10. Rubnitz JE, Razzouk BI, Lensing S, Pounds $\mathrm{S}$, Pui CH, Ribeiro RC. Prognostic factors and outcome of recurrence in childhood acute myeloid leukemia. Cancer. 2007;109(1):157-163.

11. Bose P, Vachhani P, Cortes JE. Treatment of Relapsed/Refractory Acute Myeloid Leukemia. Curr Treat Options Oncol. 2017;18(3):17.

12. Moore AS, Kearns PR, Knapper S, Pearson $\mathrm{AD}$, Zwaan CM. Novel therapies for children with acute myeloid leukaemia. Leukemia. 2013;27(7):1451-1460.

13. Tasian SK, Pollard JA, Aplenc R. Molecular therapeutic approaches for pediatric acute myeloid leukemia. Front Oncol. 2014:4:55.

14. Hinman LM, Hamann PR, Wallace R, Menendez AT, Durr FE, Upeslacis J. Preparation and characterization of monoclonal antibody conjugates of the calicheamicins: a novel and potent family of antitumor antibiotics. Cancer Res. 1993;53(14):3336-3342.

15. Sievers EL, Appelbaum FR, Spielberger RT, et al. Selective ablation of acute myeloid leukemia using antibody-targeted chemotherapy: a phase I study of an antiCD33 calicheamicin immunoconjugate. Blood. 1999;93(11):3678-3684.

16. Maniecki MB, Hasle H, Bendix K, Moller HJ. Is hepatotoxicity in patients treated with gemtuzumabozogamicin due to specific targeting of hepatocytes? Leuk Res. 2011;35(6):e84-e86.
17. Zwaan CM, Reinhardt D, Corbacioglu S, et al. Gemtuzumab ozogamicin: first clinical experiences in children with relapsed/refractory acute myeloid leukemia treated on compassionate-use basis. Blood. 2003;101(10):3868-3871.

18. Zwaan CM, Reinhardt D, Zimmerman M et al. Salvage treatment for children with refractory first or second relapse of acute myeloid leukaemia with gemtuzumab ozogamicin: results of a phase II study. $\mathrm{Br}$ J Haematol. 2010;148(5):768-776.

19. Brethon B, Yakouben K, Oudot C, et al. Efficacy of fractionated gemtuzumab ozogamicin combined with cytarabine in advanced childhood myeloid leukaemia. B J Haematol. 2008;143(4):541-547.

20. ClinicalTrials.gov. Expanded Access/ Compassionate Use Protocol For Relapsed Or Refractory CD33 Positive AML Patients In The USA Without Access To Comparable Or Alternative Therapy (AML). 2017 [cited Jan 2018]; Available from: https://clinicaltrials.gov.

21. Creutzig U, Zimmermann M, Ritter J, et al. Treatment strategies and long-term results in paediatric patients treated in four consecutive AML-BFM trials. Leukemia. 2005;19(12):2030-2042.

22. Creutzig U, Zimmermann M, Lehrnbecher $\mathrm{T}$, et al. Less toxicity by optimizing chemotherapy, but not by addition of granulocyte colony-stimulating factor in children and adolescents with acute myeloid leukemia: results of AML-BFM 98. J Clin Oncol. 2006:24(27):4499-4506.

23. Creutzig U, Zimmermann M, Dworzak M et al. Study AML-BFM 2004: Improved Survival In Childhood Acute Myeloid Leukemia without Increased Toxicity. Blood. 2010;116(21):181.

24. Creutzig U, Zimmermann M, Dworzak $\mathrm{MN}$, et al. The prognostic significance of early treatment response in pediatric relapsed acute myeloid leukemia: results of the international study Relapsed AML 2001/01. Haematologica. 2014;99(9):14721478
25. US Department of Health and Human Services. Common Terminology Criteria for Adverse Events (CTCAE) Version 4.03 2010 [cited August 2016]; Available from: http://ctep.cancer.gov.

26. Davila J, Slotkin E, Renaud T. Relapsed and refractory pediatric acute myeloid leukemia: current and emerging treatments. Paediatr Drugs. 2014;16(2):151-168

27. Arceci RJ, Sande J, Lange B, et al. Safety and efficacy of gemtuzumab ozogamicin in pediatric patients with advanced CD33+ acute myeloid leukemia. Blood. 2005; 106(4):1183-1188.

28. Aplenc R, Alonzo TA, Gerbing RB, et al Safety and efficacy of gemtuzumab ozogamicin in combination with chemotherapy for pediatric acute myeloid leukemia: a report from the Children's Oncology Group. J Clin Oncol. 2008;26(14):2390-3295.

29. Brethon B, Auvrignon A, Galambrun C, et al. Efficacy and tolerability of gemtuzumab ozogamicin (anti-CD33 monoclonal antibody, CMA-676, Mylotarg) in children with relapsed/refractory myeloid leukemia. BMC Cancer. 2006;6:172

30. Roman E, Cooney E, Harrison L, et al. Preliminary results of the safety of immunotherapy with gemtuzumab ozogamicin following reduced intensity allogeneic stem cell transplant in children with CD33+ acute myeloid leukemia. Clin Cancer Res. 2005;11(19):7164-7170.

31. Satwani P, Bhatia M, Garvin JH, Jr., et al. A Phase I study of gemtuzumab ozogamicin (GO) in combination with busulfan and cyclophosphamide (Bu/Cy) and allogeneic stem cell transplantation in children with poor-risk CD33+ AML: a new targeted immunochemotherapy myeloablative conditioning (MAC) regimen. Biol Blood Marrow Transplant. 2012;18(2):324-329.

32. Parigger J, Zwaan CM, Reinhardt D, Kaspers GJ. Dose-related efficacy and toxicity of gemtuzumab ozogamicin in pediatric acute myeloid leukemia. Expert Rev Anticancer Ther. 2016;16(2):137-146. 